Raddi, Giusepps, 1770-1829.

Crittogame brasiliane, 1822. 

Rass Raddo
rittagine hoc iliene 



\title{
GRIT TOGAME BRASILIANE
}

\author{
RACGOLTE E DESCRITTE
}

DAL SIGNOR GIUSEPPE RADDI

$\begin{array}{llllllll}M & \mathrm{E} & \mathrm{M} & \mathrm{O} & \mathrm{R} & \mathrm{I} & \mathrm{A}\end{array}$

INSERITA NEL TOMO XIX. DEGLI ATTI

D E I 2 A

SOC I E TÁ I TAL I A N A

DE L L E S C I E N Z E

RESIDENTEIN MODENA

MODENA

DALLA SUGIETÀ TIPOGRAFICA

$\operatorname{mocos} \times 8 x$ 

C.R I T T O G M E B RASILIA N E

\author{
KAGCOLTE E DESGRITTE

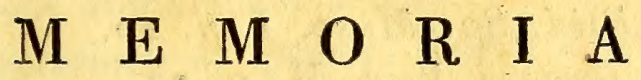 \\ $D A L S I G N O R$ \\ G I U S E P P E R A D D I
}

Sperando l' Autore della presente Memoria di potere in breve dar principio alla pubblicazione delle Tavole esprimenti le Figure delle nuove specie di Felci da esso già pubblicate con la di lui Synopsis Filicum brasiliensium, egli è per questo motivo, che il medesimo si propone di dare al seguito di esse anche quelle di tutte le specie nuove di Muschi, Licheni ec., che qui si trovano registrate e descritte.

I. SPHAGNUM magellanicum: trunco erecto subdiviso, ramulis alternatim fasciculatis deflexis, aliis brevissimis incrassatis, aliis longissimis filiformibus, foliis oblongis concavis obtusiusculis. Brid. Musc. rec. II. p. 24. tab. 5.f. I?

Trovasi negl' Acquitrini delle Vallate nella così detta Serra d' Estrella.

2. OCTOBLEPHARIUM albidum: foliis e subvaginante basi apiculato lingulatis, crassiusculis albidis patulis; theca ovata erecta. Hedw. crypt. III. p. 15. t. 6. A.

Bryum albidurn, antheris erectis; foliis lingulatis obtusis patulis. Lin. Sp. pl. p. 1583.

Bryum nanum: lariginis foliis albis. Dill. H. Musc. $p$. 364.t. 46. f. 21 .

Trovasi su i tronchi degl'Alberi nelle vicinanze di Riojaneiro, dove vi forma delle espansioni larghissime, e altrettanto dense quanto quelle del nostro Dicranio glauco.

3. DICRANÜM megalophyllum : caule adscendente ra- 
moso, foliis densissime confertis, canaliculato - subulatis subsecundis maximis. Brid. Musc. Rec.Suppl.P.IV.pag. 67.n..$^{\circ} 6 \mathbf{1}$.

Sphagnum iridans, trunco erecto diviso, foliis densissime imbricatis secundis longissimis canaliculato-subulatis diversicoloribus. Brid. Sp. Musc. I. p. 20.

Sphagnum javense. Brid. II. p. 27.

Sph. clandestinum. Palis. Beauv. Mus. p. 88.

Trovasi sulla terra umida framezzo i cespugli delle Piante sulla spiaggia marittima prossima a Rio di janeiro, e segnatamente sotto $S$. Teresa.

4. HOOKERIA pendula: caule decumbente ramoso, ramis pinnatis curvatis compressis, foliis undique imbricatis ovatis basi binervibus, capsula ovata nutante, operculo conico rostrato, calyptra carnosa pilosa basi fimbriata. Hook. Musc. exot. tab. 53.

Trovasi su i tronchi degli Alberi nelle vicinanze di Tijucca, circa a una mezza giornata di distanza da Rio-janeiro.

5. ORTHOTRICHUM longirostrum: caule repente, ramis erectis, foliis lanceolato-acuminatis striatis siccitate tortis, seta longa, capsula ovali-elliptica sulcata, calyptra glabra multifida, ciliis nullis, dentibus 16 vix per paria approximatis. Hook. exot. tab. 25.

6. ORTHOTRICHUM Swainsoni: caule repente ramis erectis, foliis ellipticis longitudinaliter plicatis, nervo breviter excurrente, seta longiuscula, capsula ovata laevi, calyptra nuda sulcata longe multifida. Hook. exot. $t$. 127 .

7. ORTHOTRICHUM rugifolium: caule repente, ramis erectis, foliis ellipticis substriatis rugosis nervo breviter excurrente, seta longiuscula, capsula ovato-oblonga laevi, calyptra campanulata nuda glabra basi 4.dentata.Hook. exot. $t$. 128.

Questa, e le due precedenti specie le ho ritrovate sopra i tronchi di diversi alberi sulla Montagna prossima a Rio-janeiro denominata il Corcovado.

8. SCHLOTHEIMIA viticulosa: caule repente, ramis 
erectis, subsimplicibus, longiusculis; foliis lanceolatis, medio canaliculatis, undulatis, patulis, siccitate tortis; sporangiis ovato-cylindraceis; calyptra glabra pilosiusculoque, multifida: $N o b$.

an Orthotrichum apiculatum Hook.??

L'abito esteriore di questo Musco è quello dell' $H_{y p-}$ num viticulosum Lin., dal quale si scosta però essenzialmente per la forma dei suoi cappuccj (calyptrae). Il suo caule è serpeggiante, e manda di tratto in tratto dei gruppetti di radicelle rossastre per mezzo delle quali si attacca alla scorza degl' Alberi su cui trovasi : i rami sono ordinariamente semplici, e lunghi da un pollice fino ad un pollice e mezzo; alle volte questi sono un pochettino ramosi. Le foglie sono lanceolate, alquanto scanalate nel mezzo, ondulate nei margini, munite di un nervo o costola assai rilevata nella lor parte inferiore e attortigliate allorchè in istato di siccità. Le cassule o sporangj sono erette, longitudinalmente angolate, atteruate alla loro base, e sostenute da delle sete rossastre lunghe $3-4$ linee, le quali, sebbene terminali, sembrano provenire dai lati dei rami, mediante l'accrescimento o rinnovazione di quest'ultimi. Il peristoma interno è una membrana reticolata irregolarmente divisa in più lacinie o denti d'una non ordinaria bianchezza. L'operculo è alquanto conico, e lungamente acuminato Il cappuccio è parimente conico e acuminato, ordinariamente glabro, raramente munito di qualche pelo e diviso alla sua base in più lacinie; egli è altresi color di seta cruda, e ferrugineo nella sua punta o estremità.

Trovasi negl'ombrosissimi Boschi del Corcovado.

9. PTERIGYNANDRUM fulgens: longum pendulum, ramorumque foliis carinatis, distichis, nitidis: theca ovata, operculo acuminato tecta. Hedw. Musc. Frond. IV.p. $10 \mathrm{I}$. t. 39. Swartz Fl. ind. occ. $111 . p .177^{6}$.

Trovasi in quantità nelle ombrosissime selve delle Montagne d' Estrella. 
10. LASIA orthotrichoides: caule repente ramosissimo, foliis lanceolato-acuminatis carinatis imbricato-patulis, pedunculis axillaribus lateralibusque, capsulis erectis ovato-cylindraceis, ore subcoarctata. Nob.

Trovasi questo Musco sopra $\mathrm{i}$ tronchi degli Alberi nelle vicinanze di Mandiocca. Il suo caule vi è serpeggiante, e da esso sorgono moltissimi rami eretti, i quali inordinatamente si dividono in molti altri ramoscèlli più o meno lunghi. Le foglie sono imbricate, ma però distanti dal caule e dai suoi rami per quasi due terzi della loro lunghezza; sono altresi lanceolate, acuminate, carinate e intere nel margine. Le foglie pericheziali son più lunghe delle cauline, e linearilanceolate: il nervo si delle une, come delle altre è sempre un poco rossastro, e termina con la loro punta o estremità. Le cassule, o sporangj, sono erette, quasi cilindriche, liscie e alquanto ristrette alla loro apertura : l'operculo è quasi conico, e terminato da una breve punta alquanto ottusa. II cappuccio (calyptra) è anch' esso conico, acuminato, ricuoperto di peli tutti diretti o voltati verso la sua punta, e diviso alla base in $5-8$ divisioni piuttosto profonde, le cui lacinie sono come troncate, e più o meno dentellate. Le sete che sostengono le cassule nascono ordinariamente dalle ascelle o inforcazioni dei rami, e spesse volte ancora dai lati di quest' ultimi; sono circa due linee di lunghezza, o anche di vantaggio, e dello stesso colore delle cassule, cioè rossastre.

I. PTEROGONIUM myurum: caule (fructifero) suberecto pinnatim ramoso, foliis undique imbricatis erectis ovatis valde concavis enervibus integerrimis, seta elongata, capsula oblonga erecta, operculo acuminato. Hook. Musc. exot. tab. 148 ?

Ritrovato nelle ombrose selve di Mandiocca sul tronco d'una Swartzia.

12. NECKERA undulata: trunco pinnatim diviso ramoso, foliis truncatis undulatis, bifariam imbricato-patentibus; 
thecis rameis, posticis, immersis. Hedw. Crypt. III. p. $5 \mathrm{I}$. tab. 21 .

Sugl' alberi delle Montagne $d$ 'Estrella.

13. PILOTRICHUM scabrisetum: caule repente, ramis subsimplicibus erectis, foliis distichis ovato-lanceolatis concavis dentatis binervibus, pedunculis scaberrimis capsulâ cylindricâ subinclinata. Brid. Suppl. P.IV. pag. I4I.

Neckera scabriseta: subramosa, foliis distichis lato lanceolatis subdimidiatis binervibus dentatis, setâ exertâ scaberrima. Schwägr. Suppl. I. P. II. p. 153. tab. 82.

$\mathrm{Gl}^{\prime}$ individui da me ritrovati sulle Montagne d' Estrella differiscono soltanto dalle citate descrizioni Brideliana e Schwägricheniana, per la quasi mancanza assoluta dei denti nelle foglie, e convengono poi esattissimamente in tutto il resto. ( an. Sp. nova? )

14. LESKEA rotulata: trunco e summitaté ramoso, foliis acute cordatis bifariam imbricatis inferne tectis, sporangiis oblongis pendulis basi rugulosis. Hedw. Sp.Musc. p. 2 I 3.t. 5 I. fig. 8 - 13.

Leskia rotulata; caule erecto summitate diverse ramoso, foliis distichis acute cordatis serratis, tegminibus integerrimis, perigonialibus dorso pilosis, cápsula oblongâ pendulâ. Brid. Musc. rec. Suppl. P. II. p. 48.

Abita le Montagne d'Estrella, dove ordinariamente si trova a piè degli Alberi, ed anche sulla nuda terra presso i Torrenti.

15. LESKEA mollis : flexuose pendula, ramis compressiusculis pedunculisque brevibus. Hedw. Crypt. IV. p. 103.t. 40 .

Quasi tutti gl' Alberi delle cupe selve, che rivestono le Montagne d'Estrella son ricuoperti di questa specie di musco, il quale pende lungo i loro rami a somiglianza dei Licheni filamentosi, e la di cui lunghezza giunge sovente a due piedi circa.

16. RACOPILUM tomentosum. Pal. Beauv. Brid. Musc. rec. suppl. P. IV. p. 152. 
Hypnum tomentosum; trunco repente, ramis vagis, erestiusculis; foliis ovato-lanceolatis, apiculatis, bifariam patentibus, accessoriis minimis, dissitis, aristatis; thecis cernuis, operculo rostrato. Hedw. Musc. frond. IV. p. 48. tab. 19.

Hypn: tomentosum; surculo repente vage ramoso; foliis distichis, ovato-lanceolatis, apiculatis, accessoriis minimis aristatis; capsulae cernuae, operculo oblique rostrato. Brid. Musc. rec. V. II. P. II. p. I 30. Suppl. P. II. p. 88.

Hypn. tomentosum. Swartz. Fl. ind. occ. Prodr. p. 141.

Trovasi sulle ombrose rupi delle Montagne d'Estrella.

17. HYPNUM spiniforme : surculo erecto simplicissimo; foliis denticulatis ; pedunculis basilaribus ; capsulae ovatae cernuae, operculo oblique rostellato. Brid. Musc. rec. II. P. II. p. 49. Hedw. Musc. frond. III. p. 59. t. 25.

Hypn. spiniforme; fronde simplicissimâ, foliolis patentibus, subulatis, pedunculis radicalibus. Lin. Suppl. p. 1587 .

Trovasi a piè degli Alberi, ed anche sui Massi, nelle ombrosissime selve delle Montagne d'Estrella.

18. HYPNUM circinale: caule repente dense pinnatim ramoso, foliis lanceolato-subulatis subserratis secundis circinalibus enervibus, seta breviuscula, capsula ovata cernua, operculo conico. Hook, exot. tab. 107.

Trovasi come sopra.

19. HYPNUM imbricatum, pendulum vage ramosum, foliis quinquefariam imbricatis ovatis enervibus laevibus fornicatis mucronulatis. Schiwägr. Suppl. I. P. II. p. 209.

Hypn: pentastichum. Brid. Musc. rec. suppl. P. IV. p. 166.

Trovasi sulle Montagne sopraindicate, dove però è rarissimo.

20. HYPNUM patulum: surculis filiformibus repentibus, ramulis brevissimis, foliis trunci semivaginantibus patulis, ramulorum vagis reflexis lanceolatis: ductulorum fasciculo in apicem filiformem exeunte. Schwägr. 279, $t .73, f .1$.

Trovasi sugl' Alberi nelle vicinanze di Mandiocca. Non conosco punto la sua fruttificazione. 
21. HYPNUM? longissimum: caule repente, filiformi, ramis sesquipedalibus et ultra; subflexuosis, pendulis, ramulis vagis, abbreviatis; foliis lanceolatis, divaricato-patulis, integerrimis, apice in mucronem longissimum terminatis. Nob.

Non ostante la prodigiosa quantità di questo Musco da me incontrata sulle Montagne d'Estrella, non mi fu possibile poterne rintracciare alcun' individuo con la fruttificazione. Vi è, come la Leskea mollis, pendente ai rami degl' alberi a somiglianza dei Licheni filamentosi, e le sue principali diramazioni non di rado sorpassano un piede e mezzo di lunghezza .

22. HYPNUM? ... caule vage ramoso vel ramoso-subdichotomo gracili, foliis lanceolatis longe acuminatis divaricato-patulis integerrimis, perichaetiis lateralibus numerosissimis. Nob.

Ritrovato presso la Montagna denominata Corco-secco. Non conosco le sue Gassule mature.

23. POLYTRICÜM glabrum: trunco simplici, foliis lineari lanceolatis integerrimis carina laevibus membrana reducta carentibus, capsulae tetraedrae operculo e basi plana mucronato. Brid. Musc. rec. II. p. 82.

Polytr. glabrum, simplex, foliis erectiusculis planis integerrimis apice scabriusculis, teca appendiculata parallelepipeda. Schwägr. Suppl. I. P. II. pag. 311. Ritrovato sulla Montagna detta do Frade.

24. CATHARINEA pseudo-polytrichum: trunco subramoso, foliis subulatis canaliculatis serratis, sporangiis ovatooblongis subcernuis, peristomatis dentibus 64. inflexis albicantibus, opercula e basi conica in rostrum longum acuminatum incurvum educta. Rad. Act. Soc. Ital. Sc. XVIII. p. 33. tab. 4. fig. 3.

Catharinaea magellanica. Brid. Musc. rec. Suppl. P. IV. pag. 204.

Polytrichum magellanicum: foliis lineari-lanceolatis, acutis, denticulato-serratis, capsula oblonga subcylindrica ere- 
10

ctiuscula, calyptra simplici. Menzies Act. Soc. Lin. 4.p.71. t. 6. fig. 1. Schwägr. Suppl. I. P. II. p. 332.

Pogonatum magellanicum. Palis. Beauv. Aetheo. 84.

Trovasi abbondantemente sulle Montagne che costituiscono la Serra do Frade.

Schulthesia. Nuovo genere da me stabilito in onore del meritissimo e dottissimo Amico Sig. L. Schulthes Professore di Botanica nella Università di Zurigo, i di cui Caratteri generici sono: Calyx subcompressus, angulato-plicatus, ore laciniato; Corolla monopetala membranosa limbo varie secto; Capsula subrotunda 4-valvi; spiculis oblongis ovalibusque ab imbricatis squarnis convexis effectis, in quibus organa masculina (Antherae) includuntur.

25. SCHULTHESIA brasiliensis : caulibus procumbentibus, adscendentibus, ramosissimis, ramis orizontalibus; foliis dimidiato-ovatis, subrecurvis, bifariam imbricatis, integerrimis subtus amphigastriis denticulatis triplici serie dispositis, lateralibus lanceolatis obtusis, intermediis oblongis; calycibus angulato-plicatis, crispis. Nob.

Ho ritrovato questa pianta nelle ombrosissime foreste delle Montagne d'Estrella sopra i tronchi degl' alberi. Il suo abito esteriore è precisamente quello dell' Antoira vulgaris (jung. platyphylla Lin.). Le sue foglie però sono a metà ovate, e un poco recurve, ovvero falcicolate; sono superiormente convesse, e interissime nel loro margine. Le foglie pericheziali, quelle cioè situate alla base dei Calici sono dentato-ciliate nel bordo, e più grandi delle altre. I Calici sono appena un poco compressi ; hanno dieci angoli longitudinali ondulatocrespi, e sono alquanto ristretti nella loro estremità. Questa, per dar passaggio alla cassula o sporangio allor che è matura, si apre ora in tre, ora in più lacinie, sempre però negli angoli. Le cassule son quasi rotonde; le loro valve ovali, o ovato-lanceolate. Tutta la parte inferiore del caule e dei rami è ricuoperta da tre ordini di stipole (amphigastria) embriciate, ovvero soprapposte l'una sopra l'altra, le quali nei 
due ordini o ranghi laterali sono lanceolate e ottuse, oblongate e più grandi in quello di mezzo, tutte però munite di piccolissimi denti più o meno radi nel loro margine. Delle spighette bislunghe, che posano sull' estremità di altrettanti cortissimi ramoscelli muniti ciascuno di $4-6$ foglie, e situate sopra individui distinti, racchiudono entro le loro squarnme dei corpiccioli rotondi (antherae) costituenti l'organo maschile di questa pianta.

FRULLANIA (ved. Jungermanniografia etrusca p. 9. ) 26. FRULLANIA dichotoma: caulibus dichotomis, longissimis, pendulis; foliis ovato-acuminatis, bifariam imbricatis, auriculis minutis conformibus; amphigastriis quadrato oblongis, dentatis; calycibus laevigatis, involutis. Nob.

I cauli di questa bella e straordinaria jungermannoidea sono ordinariamente lunghi circa un piede e mezzo, ed anche di vantaggio; sono altresi decisamente dicotomi, e alquanto divaricate le loro diramazioni. Le foglie sono ovatoacuminate, interissime, un poco flessuose nel loro margine inferiore, e munite di piccolissime orecchiette quasi della stessa figura delle foglie. Un ordine di stipole quadrilunghe, dentate nel loro estremo margine e soprapposte l' una sull'altra a somiglianza degl'embrici dei tetti, cuoprono interamente tutta la parte inferiore del caule: le foglie pericheziali sono più grandi, e dentate nei loro margini. I calici sono allungati, triangolari, lisci nella lor parte superiore, e con i lati o angoli laterali alquanto accartocciati per il lato inferiore di maniera che, nel loro stato di siccità, compariscono quasi rotondi. Le cassule sono perfettamente simili a quelle delle Frullanie maggiore e minore ( jung. tamarisci, e jung. dilatata Lin.), e le sete che le sostengono appena oltrepassano di un pochettino la lunghezza del calice.

Trovasi come la precedente, nelle ombrosissime selve delle Montagne d'Estrella, sempre pendente ai rami degli alberi.

27. FRULLANIA filicina: caule repente, ramis adscen- 
dentibus vel erectiusculis, pinnatis bipinnatibusque, ramulis acutis; foliis ovatis apice serrulatis, subtus auriculatis, auriculis minutis sabrotundatis; amphigastriis ovato-quadratis dentatis; calycibus laevibus. Nob.

Jungermannia filicina. Swartz FI. ind. occ. III. pag. 1866. Jung. ( filicina) caule erecto, pinnatim ramoso, foliis inaequaliter bilobis, lobis superioribus majoribus ovatis acutiusculis apice serratis; inferioribus minutis, subrotundatis, conduplicatis; stipulis obovato-quadratis, serratis, calycibus latera libus oblongo-obcordatis, apiculatis. Hook. Musc. exot.t. 142.

Trovasi con la precedente.

28. FRULLANIA brasiliensis: caulibus repentibus, ramosissimis; foliis bifariam imbricatis vel distichis, subrotundis, convexiusculis, integerrimis, subtus auriculatis, auriculis acute-lanceolatis, amphigastriis oblongiusculis, emarginato bifidis, laxe imbricatis; calycibus obsolete triangularibus vel cilyndraceis, laevibus. Nob.

Trovasi frequentemente sulle Montagne suddette, ed anche sul Corcovado.

Il suo abito esteriore è quello della Frullania minore ( jung. tamarisci Lin.), con la quale conviene perfettamente nella grandezza, nella diramazione dei cauli e nella figura delle sue foglie; nella nostra però sono queste inferiormente munite d' una picciola orecchietta acutamente lanceolata, mentre nell' altra è rotonda, disgiunta dalle foglie, e per conseguenza una vera stipola. Le stipole di mezzo sono oblongate, emarginato-bifide, un poco rovesciate nei lati, e molto grandi : esse ricuoprono interamente la parte inferiore del caule, e de' suoi rami. I calici mostrano appena un poco nell' estremità d' esser triangolari, essendo quasi cilindrici verso la base; sono interamente levigati; e la punta con la quale terminano le loro lacinie è altrettanto lunga, che nella Frullania maggiore (jung. dilatata Lin.), o fors' anche più. Le cassule, egualmente che la lunghezza delle sete che le sustengono, sono precisamente simili, come lo sono an- 
cora le spighettine, che trovansi sopra individui distinti e che contengono 'l' organo maschile di queste Piante.

Frullanoides. Le specie di questo genere non differiscono da quelle del precedente, che per avere un angolo di più i loro calici. Presentano esse i seguenti caratteri generici : Calyx compresso-quadrangularis, ad extremitatem in 4 . lacinias rotundas et apiculatas divisus: superiori parte plano-convexa, et sulcato-plicata. Corolla monopetala limbo varie secto. Capsula vel sporangio ovato-subrotundo in quatuor valvas aequales dehiscens.

29. FRULLANOIDES Rio-janeirensis : caulibus repentibus, ramosissimis; foliis bifariam imbricatis, subrotundis, convexiusculis, integerrimis, subtus auriculatis; auriculis ventricosis; stipulis amplissimis, emarginato-bifidis. Nob.

Trovasi sugl'alberi nei boschi del Corcovado, Montagna prossima a Rio-janeiro.

Il suo abito è presso a poco quello della precedente specie. Le sue foglie però sono più grandi, fornite alla loro base d' un' orecchietta, la quale, dopo essersi ripiegata entro la parte concava delle medesime, vi forma un corpo ventricoso, e semisferico. Le stipole sono assai grandi, un poco più larghe che lunghe, emarginate, convesse nel loro centro, e la più gran parte di esse forate da una radicella piuttosto grossa, la quale va ad impiantarsi nella scorza del1' albero su cui essa si trova. I calici sono compresso-quadrangolari, il cui lato superiore è piano-convesso, con un solco longitudinale nel mezzo, ai lati del quale si vedono due ripiegature o linee salienti, che parallelamente scorrono quasi tutta la lunghezza del Calice; gli altri tre lati sono profondamente concavi, e tutti tre insieme formano la parte inferiore di esso. L' estremità di questi calici si apre negli angoli in quattro lacinie rotondate, e terminate ciascuna da una cortissima punta, come appunto nella Frullania minor. Le cassule, egualmente che le sete che le sostengono, sono parimente come in quest' ultima. 
3o. FRULLANOIDES densifolia: caulibus procumbentibus repentibusque, ramoso-dichotomis; foliis distichis vel bifariam et dense imbricatis, subrotundo-ovatis, integerrimis, subtus auriculatis, auriculis minutis foliis arcte adpressis; stipulis latissimis, integris. Nob.

Il caule di questa pianta è ramoso-dicotomo, e della lunghezza quasi $d$ ' un pollice. Le sue foglie sono quasi rotonde, o rotondo-ovate, intere nel margine, densamente imbricate, assai convesse, e munite alla loro base d'una piccola orecchietta strettamente aderente alla parete interna della foglia, di cui sembra piuttosto una piccola ripiegatura. Le stipole sono molto più larghe che lunghe, intere e alquanto rovesciate nel margine, e, come le foglie, fittamente imbricate: qualche volta presentano alcune di esse un leggiero indizio di smarginatura. I calici son perfettamente simili a quelli della precedente specie. Trovasi com' essa sugl' alberi negl' ombrosi boschi del Corcovado.

$C A N D O L L E A$, (ved. jungermanniografia etrusca p. I1.) 31. CANDOLLEA simplex. Nob.

Jungermannia simplex, surculis repentibus, partialibus erectis indivisis, simpliciter foliatis, foliis remotiusculis, ovatis serrato-ciliatis. $S_{w} . F l$. ind. occ. pag. 1848. Prodr. 143.

32. CANDOLLE A diffusa. Nob.

Jung. diffusa, surculis ramosissimis divaricatis dichotomis; foliis imbricatis ovatis acutis; accessoriis subrotundis; apice retusis dentatis. $S_{W} . F l$. ind.occid. p. 1860. Prod. 144.

33. Candollea adiantoides. Nob.

Jung. adiantoides, surculis erectis subdivisis, simpliciter foliatis; foliis dimidiato-ovatis, denticulato-ciliatis; fructificationibus axillaribus. Swarz $F l$. ind. occ.p. 1842. Prod. 142.

Jung. adiantoides, caule repente, ramis erectis divisis, foliis distichis horizontalibus dimidiato-ovatis denticulato-ciliatis, calyce terminali urceolato, ore compresso fimbriato; seta brevi. Hook. exot. tab. 90.

Jung, annotina. Menz MSS. 
34. CANDOLLEA cristata. Nob.

Jung. cristata, surculis repentibus, partialibus e rectiusculis subdivisis simpliciter foliatis; foliis incumbentibus dimidiato-cordatis lanceolatis antice denticulatis, subtus lobis conniventibus cristatis. Swartz Fl. ind.oce. p. 1845 . Prod. 143.

35. CANDOLLEA repanda. Nob.

Jung. repanda, caule repente ramoso, ramis erectis, foliis exacte distichis arcte imbricatis horizontalibus oblongoovatis planis, basi insigniter decurrentibus omnino integerrimis. Hook. exot. t. 86.

Le cinque sopra indicate Candollee trovansi tutte sulle Montagne d'Estrella, egualmente che su quelle prossime a Rio-janeiro. La più comune è l'adiantoides, della quale credo esser le altre altrettante varietà.

36. CANDOLLEA complanata: caulibus repentibus, ramosis, complanatis; foliis rotundatis imbricatis, planis, integerrimis, auriculis subromboidalibus, foliis arcte adpressis; calycibus lateralibus terminalibusque, numerosissimis. Rad. jung. Etr. $p . \mathrm{I}^{3}$.

Jung. complanata. Lin. ec.

Il Corcovado è il solo luogo dove ho trovato questa specie, la quale vi è piuttosto rara.

37. JUNGERMANIA pallens: surculis erectis ramosis simpliciter foliatis, foliis cordato subrotundis integris, subtus basi complicatis; perianthiis monophyllis, retusis, infundibuli formibus. $S_{W} . F l$. ind. occ. p. 1847. Prod. 143.

Questa, e le sei seguenti specie trovansi tutte sulle diverse Montagne, che costituiscono la così detta Serra do Frade.

Jungermannia prostrata; surculis simpliciusculis prostratis repentibus, simpliciter foliatis ; foliis subrotundo-ovatis integris marginatis. Swartz fl. ind. occ. III. p. 1846. Prod. 142.

38. JUNGERMANNIA brasiliensis: caule prostrato, simpliciusculo; foliis patentibus, oblique semiamplexicaulibus, subquadratis, concavis, extremitatibus profunde emarginatis 
seu emarginato-bidentatis; amphigastriis bifidis, laciniis dentatis; calycibus terminalibus oblongis, ore laciniato. Nob.

Il caule di questa jungermannia è della lunghezza di due linee fino a mezzo pollice, ed è in quest' ultimo grado d'accrescimento, che alle volte presenta qualche piccola diramazione. Lungo la parte inferiore del medesimo trovansi framischiate da numerose e tenuissime radicelle delle stipole ( amphigastria ) bifide, ovvero divise fin quasi alla base in due lacinie, le quali hanno ciascuna nel loro bordo esterno due, qualche volta tre denti più o meno lunghi. Le foglie son presso a poco simili a quelle dell'jung. excisa Dicks: e i calici a quelli dell' jung. bidentata Lin. Le Gassule sono rotonde, e sostenute da dei pedicelli ( setae) della lunghezza di circa due linee.

39. JUNGERMANNIA serrulata; surculis erectis simpliciusculis subteretibus; foliis imbricatis, convexis, subsecundis, cordato-subrotundis accessoriisque bidentatis serratis. Swartz fl. ind. occ. III. p. 1854 .

Jung. serrulata, caule erecto dichotomo, foliis distichis v. secundis subrotundis subconduplicatis spinuloso-dentatis apice emarginatis, stipulis magnis rotundatis convexis spinuloso-dentatis apice emarginatis, calyce cylindraceo ore dentato. Hook. exot. tab. 88 .

Trovasi sovente a piè delle rupi umide, particolarmente nei cosi detti Gemitivi.

40. JUNGERMANNIA quadripartita; caule erecto subdichotome ramoso flexuoso, foliis undique insertis distantibus erecto-patulis 4-partitis, segmentis capillaribus integerrimis vel basi sublaciniatis. Hook. exot. $t$. 117 ?

4I. JUNGERMANNIA connata; surculis subdivisis diffusis, foliis oppositis incumbentibus apice retusis emarginatis, postice auricula bidentula connatis. $S_{W}$. $f$. ind. occ. III. p. 1851. Prod. 143.

42. JUNGERMANNIA tomentella; surculo erectiusculo, bipinnato: foliis bifariis, inaequaliter bilobis; lobis inferioribus 
minutis; superioribus bipartitis; utrisque apice margineque capillariter multifidis; stipulis subquadratis, laciniatis: fructu axillari; calycibus oblongis cylindraceis, hirsutis; ore aperto. Hook. brit. jung. tab. 36.

43. JUNGERMANNIA serpillifolioides: caulibus procumbentibus subramosis; foliis distichis, alternatim imbricatis, rotundis, integerrimis exauriculatis; stipulis rotundatis, emarginatobifidis. Nob.

Differisce dall' jung. serpillifolia Dicks. per le sue foglie decisamente rotonde e affatto mancanti d'orecchiette, per le sue stipole più grandi e più fitte che in quella, e per esser meno o quasi punto ramosa. Non conosco la sua fruttificazione.

Trovasi sulla nuda e umida terra framezzo i cespugli delle piante sulla spiaggia marittima prossima a Rio-janerio.

44. JUNGERM ANNIA serpillifolia; surculo repente, filiformi, flexuoso, vagè pinnatim ramoso: foliis distichis, auricularibus, ( seu sub bilobis; lobis inaequalibus, superioribus majoribus, rotundatis, basi subtus ventricosis; inferioribus minutis, involutis:) stipulis rotundatis, acutè profundèque bifidis; fructu laterali axillarique; calycibus latè obovatis, pentagonis; ore contracto, elevato, subdentato. Hook.Brit.jung. tab. 42.-Dicks. Crypt. Plant. Fasc. 4. p. 19.-Rad.jung. etrusca p. 24.t. $V . f$. I.-Mich. Nov. pl. gen. 9. tab. 6. f. I9.

E comunissima sulle Montagne d'Estrella, dove trovasi sugl' alberi, egualmente che sulla terra'.

Metzgeria ( ved. jung. etrusca p. 34.)

45. METZGERIA ciliata: frondibus lineari-ramoso-dichotomis, subdivaricatis, costatis, margine ciliatis; calycibus turbinatis, piloso-hirtis. Nob. an var. Metz. glabrae. (jung. furc. Lin.). Hedw. Theor, generat, t. $2 \mathrm{I}, 22$ ?

Trovasi sugl' alberi nei 'Boschi presso Mandiocca.

Le sue frondi son ramoso-dichotome, membranacee, piane, lineari, costantemente contornate nel loro margine da fitti peli bianchi e trasparenti, alquanto ottuse nelle estre mità, larghe quasi una mezza linea, e lunghe da un polli- 
ee fino a un poilice e mezzo: scorre nel loro centro un pic. col nervo o costola, che dalla base va fino alle estreme diramazioni, ricuoperto nella parte inferiore di esse di numerosi e fitti peli bianchi perfettamente simili a quelli che circondano il margine. I calici hanno origine dalla parte inferiore del nervo suddetto il più delle volte nelle ascelle delle sue diramazioni; quindi si piegano ai lati della fronda per prendere una direzione verticale al di sopra del piano di esse, e fin dalla loro inserzione; sono interamente ricuoperti di numerosi e fitti peli bianchi, compressi e ottusi simili ai già sopra descritti ; sono altresi della stessa grandezza e figura, che quelli della Metzgeria glabra (jung. furcata I.i川.), come lo sono ancora le cassule, egualmente che i globet:i, che non di rado osservansi irregolarmente sparsi sopra la superficie delle frondi sterili. Le diramazioni delle frondi sono più allungate, e più regolarmeute dicotome, che nella jung. furcata Lin.

Viviania, nuovo genere da me stabilito in onore del celebratissimo Sig. Domenico Viviani Professore di Botanica e Storia Naturale nella Reale Università di Genova, dietro i seguenti caratteri generici : Calyx duplex e pagina superiori frondis prodeunte. Corolla monopetala limbo varie secto. Capsula oblonga quadrivalvis. Antherae subrotundae squamis membranac:o-foliaceis tectae, in pagina superiori frondis distinctae.

46. VIVIANIA sinuata : frondibus adscendentibus, subramoso-dichotomis planis, margine integris pinnatifidoque-sinuatis; calycibus exterioribus profunde laciniatis, laciniis irregulariter dentatis. Nob.

Jungermannia ( sinuata) subacaulis ; frondibus lanceolatis planis subdichotomis margine integris pinnatifidoque-sinuatis. $S w . F l$. ind. occ. III. p. $187_{3}$. Prod. 145.

I calici esterni o squamme perigoniali di questa Pianta sono ( sebbene più piccoli) quasi simili a quelli dell' jung. hibernica Hook. 
Trovasi nei luoghi muscosi e umidi, d'ordinario presso i torrenti, sulle Montagne d' Estrella, dove è comunissima . Ad essa framischiate vi ho trovato ancora delle frondi sterili a margine intero, concave o quasi canalicolate, pochissimo o punto ramose, il di cui nervo è, nel maggior numero di esse, quasi interamente coperto nella sua parte superiore di minutissime e fitte squamme ora intere, ed ora terminate da due o tre piccoli denti, della stessa sostanza delle frondi, le quali racchiudono altrettanti corpiccioli (antherae) rotondi e giallastri. Le loro estremità sono lungamente attenuate, come appunto nell' jung. rhizobola Hook., ma non hanno, come in questa, il margine incrassato e dentato. Certamente appartengono esse ad una nuova e distinta specie.

47. VIVIANIA hymenophyllum: frondibus adscendentibus, subramoso-furcatis, palmato-dichotomisque, membranaceis,nervosis, subdenticulatis; calycibus superaxillaribus. Nob.

Jung. hymenophyllum; frondibus palmatis dichotomis nervosis, marginibus denticulatis, fructu in dichotomiarum axillis, calyce duplice, ext. squama exigua dentata, int. oblongo-cylindraceo ore dentato. Hook. Musc. exot. tab. 14.

Le sue frondi sterili sono or semplici, ora un poco ramose, ed or semplicemente forcate; sono altresi ottuse, oppure leggiermente smarginate all' estremità, glabre, di un verde chiaro, elegantemente reticolate allorchè osservate sotto la lente, e qualche volte munite di radi e minutissimi denticelli nel loro margine. Sopra la parte superiore di queste frondi, e precisamente lungo il loro nervo o costola, si osservano due ranghi di piccole squamme alternativamente aderenti ai lati di detto nervo, membranoso-foliacee, convesse e terminate da due o tre piccoli denti, ciascuna delle quali racchiude un corpicciolo (anthera) quasi rotondo e giallastro costituente l' organo maschile di questa pianta.

Di questa rarissima Epatica ho trovati solamente alcuni pochi individui maschi sulla Montagna detta il Frate, cosi denominata da quegl' Abitanti per motivo della sua calva e 
rotonda sommità, ch' essi assomigliano alla testa d'un Domenicano.

48. MARCHANTIA papillata: frondibus dichotomis, emarginatis, supra viridibus reticulato-areolatis, areolis vesiculoso perforatis, subtus marginibusque violaceis, capitulis vel receptaculis medio papillatis, extremitatibus radiorum complanato-dilatatis. Nob.

La Marchantia capitulo eleganter dissecto, radiis ad extremitatem complanatis, et infra cochlearis instar excavatis. Mich. n. pl.gen.2. $t$. I. $f .4$. è una mera varietà di questa nostra specie Brasiliana, dalla quale differisce soltanto per essere un poco più grande in tutte le sue parti, e per la maggior carnosità delle sue frondi, le quali sono anche alquanto sinuate nei loro margini, ciò che non è nell' altra . I filamenti articolati e compressi sparsi lungo i peduncoli che sostengono i ricettacoli sono altresi in assai maggior numero nella specie Micheliana, come ho osservato in tutti gl'individui da me ritrovati nei contorni di Firenze, particolarmente nel luogo stesso indicato. dal Micheli, sebbene ciò non apparisca dalla figura da esso esibita, nè tampoco dalla di lui descrizione. Propongo adunque di decifrare la prima col nome di Marchantia papillata $(\alpha)$ Brasiliensis, la seconda con quello di March. papillata $\beta$. italica.

49. MARCHANTIA chenopoda: frondibus ramoso-dichotomis, emarginatis, marginibus sinuatis lobatisve, superficie areolato-vesiculosis, vesiculis albidis perforatis; receptaculo communi dimidiato et lateraliter pedunculato, masculo palmato quadrifido, foemineo convexo-subcampanulato subintegro: Nob.

March. chenopoda, receptaculo communi masculo subpeltato palmato-quadrifido femineoque dimidiato, subradiato. Swartz Fl. ind. occ. III. p. 1880.

March chenopoda, capsula dimidiata palmata quadrifida. Lin. Syst. Nat. Ed. XIII. p. I 354.

March. pileo laterali palmato quadrifido, frondibus sinuosis. Lam. Dict. Enc. III. p. IIO. 
Lichen anapodocarpos. Plum: Fil. p. 143. t. 142. Tournef. I. R. H. p. 55o. Dill. H. Musc. t. 77 . f. 8.

Questa singolare Epatica trovasi in abbondanza sulle Montagne d'Estrella a piè delle rupi umide e muscose. Essa è da riconoscere facilmente a prima vista da tutte le altre specie di questo genere, mediante la straordinaria forma o figura de' suoi ricettacoli, i quali compariscono come troncati da un lato e per il medesimo aderenti ad un pedunculo proveniente dall' estremità della costola delle frondi. Nel Maschio questi ricettacoli sono piani e divisi in quattro parti o lobi diseguali in lunghezza, stati da Plumier assomigliati a un piede $d$ ' Oca, ma che in vece possono più ragionevolmente assomigliarsi a quelli di un Colimbo. Nella pianta femmina sono essi molto convessi e quasi campaniformi, interi o pochissimo divisi nel loro margine, ed hanno quattro ringrossamenti carnosi o costole, che traggono origine dal peduncolo che li sostiene, fra i quali son situate le cassule, come nella March. polymorpha. La forma di questi ultimi ricettacoli può paragonarsi, presso a poco, a quella d'uno spengitojo da Chiese. Le frondi non sono cosi profondamente, nè tanto irregolarmente sinuate quanto appare dalla Figura Plumieriana esibita da Dillenio, essendo essa molto esagerata in tutte le sue parti; hanno la loro superficie areolata, e ciascheduna areola presenta nel suo centro una vessichetta biancastra forata nel mezzo, egualmente che nei ricettacoli di ambedue i sessi; nella parte inferiore sono pavonazze, ma verdi intorno al margine.

5o. MARCHANTIA hirsuta: frondibus primordialibus simplicibus, submembranaceis, emarginatis, ex innovatione ramoso-dichotomis, marginibus undulato-sinuatis; receptaculis communibus setoso-radiatis ex apice frondis prodeuntibus. Nob.

March. hirsuta, receptaculo communi masculo sessili femineoque margine hirsuto, hemisphaerico. Swartz Fl. ind. occ. III. p. I879. Prod. 145.

Le frondi di questa bella Epatica sono in principio sem- 
plici, smarginate all' estremità, e ondulato-sinuate nei margini, dove, invecchiando, spesso si lacerano fin quasi alla loro costola; sono esse altresi quasi membranose, ovvero semimembranose e liscie nella loro superficie. Dall' estremità di queste frondi annualmente ne compariscono delle nuove simili alle prime, ed è per queste innovazioni, che esse divengono ramose e dichotome. Non conosco la fruttificazione di questa Epatica; ma l'assoluta mancanza di quel reticolo, e di quelle vessichette, che costantemente si osservano sulla superficie delle frondi di tutte le Marcanzie, mi fa supporre, che possa questa formare un genere a parte. Io vi ho solamente osservati dei corpi carnosi, schiacciati e rotondi, della figura precisamente d' una ruotella o piccola ruzzola, i quali non presentano alcuno indizio di fruttificazione, ma il celeberrimo Signor Swartz dubitò esser questi i ricettacoli che contengono l' organo maschile di questa pianta. Detti corpi sono in principio dello stesso colore delle frondi, cioè $d$ ' un verde cupo, oscuri di poi e quasi sessili; ovvero sostenuti da un pedicello di circa mezza linea, che ha origine dalla costola delle fiondi medesime, ma sempre all' estremità di esse, e nelle loro innovazioni; sono altresi contornati da lunghi e numerosi cigli o setole biancastre, schiacciate, attenuate verso la loro estremità e sovente con delle brevi diramazioni.

Trovasi in quantità nelle vicinanze di Mandiocca, come su quasi tutte le Montagne d'Estrella, sempre però vicino ai torrenti sopra i sassi muscosi bagnati dai medesimi.

51. ANTHOCEROS Brasiliensis: fronde laciniata, vel laciniato-multifida, margine inaequaliter dentato ac lacinulato crispo, raris verruculis subfuscis consperso ; capsula uno latere dehiscens. Rad. Mem. ins. nel T. XVIII. degl Atti della Soc. It. delle Scienze a p. 34. t. IV. fig. 4.

Anthoceros crispus: frondibus sinuatis laciniatis undulatis, margine crispis. Swartz Fl. ind. occ. III. p. 1884. Prod. 146 ? 
52. RICCIA glauca: frondibus furcatis, dichotomisve, reticulato-punctatis, extremitate canaliculatis, obtusiusculis; marginibus albicantibus. Rad. nov. rarior. E Dec. I. p. 4. tab. 2. $f .4$ ?

Cresce in abbondanza sulla spiaggia marittima di Riojaneiro, particolarmente sotto la Chiesa di Nossa Senhora da Gloria.

53. FUCUS natans: caule compresso, filiformi, pinnatn: ramis alternis, simplicibus : foliis lineari-lanceolatis, serratis: vesiculis sphaericis, petiolatis, petiolo plano: receptaculis cylindraceis, racemosis. Turn. Fuc. I. p. 99. $t$. 46 .

Fucus Sargasso var. I. Gm. H. Fuc. p. $9^{3}$.

Fucus natans. Lin. Sp. pl. 1620. Syst. nat. Ed. Gm. I380.

Fucus acinarius var. Esper. Ic. Fuc. I. p. 130. t. 66.

54. FUCUS bacciferus; caule tereti, filiformi, bipinnato: ramis alternis, simpliciusculis : foliis linearibus, serratis: vesiculis sphaericis, petiolatis; petiolis teretibus. Turn. Fuc. I. p. 103.t. 47:

Fucus Sargasso. Gm.H. Fuc. p. 92.

Fucus natans. Esper. Ic. Fuc. I. p. 49. t. 23.

Lenticula marina vulgaris.-Park. Thes. $128 \mathrm{I}$.

Fucus denticulatus. Forsk. Fl. Aeg. 191. Lin. Syst. nat. ed. Gm. I 38 I.

Ambedue questi fuchi li ho incontrati in una quantità prodigiosa in vicinanza del Tropico del Cancro. Nella più gran parte degl' Individui pescati di quest' ultimo, e da me osservati trovavansi aderenti una quantità di minutissime Serpule, i di cui abitatori tramandavano una viva luce fosforica, che brillava a guisa di altrettante minutissime stelle, ma che si estingueva dopo qualche minuto.

55. FUCUS flagelliformis; fronde cartilaginea, lubricâ, terete, filiformi, ramosâ ; ramis subdistichis, elongatis, simpliciusculis, nudis, truncatis: seminibus nudis, in fronde immersis, fibris immixtis. Turn. II. p. 35. t. 85. 
var $\beta$. tortilis; fronde tenui, tortuosa; caule ramis sparsis brevibus obsito.

Trovasi in abbondanza nella Baja di Rio-janeiro e segnatamente presso l' imboccatura del Rio Inhumirim.

56. ULVA undulata: frondibus aggregato- caespitosis, subramosis, viridibus; planis, extremitatibus attenuatis, margine undulatis, sinuato-denticulatis. Nob.

Trovasi aderente agli scogli battuti dall' acqua del mare lungo la costa marittima di Rio-janeiro, ove spesso vi forma delle larghissime espansioni.

Le sue frondi sono variabilissime in quanto alla loro lunghezza, e larghezza; sono altresi piane, un poco ramose, spesso ancor semplici, di un verde piuttosto cupo, ondulate nei loro margini, più o meno profondamente sinuate, e irregolarmente denticolate. La loro maggior lunghezza, da me osservata, è di circa sette in otto pollici.

an. Ulva Linza. Lyngb. Teutam. Hydroph. dan. p. 32??

57. CONFERVA lichenoides: ramosa, ramis alternis articulis diametro triplo longioribus. Nob.

Trovasi questa.singolare Conferva nelle adiacenze di Man. diocca sopra la scorza dei piccoli arboscelli, egualmente che sulle loro foglie, dove vi forma delle espansioni rotonde e semirotonde, le quali prendono l'aspetto d'un Lichene, d'onde la sua specifica denominazione. Le minute Pezize di color quasi carnicino, che di sovente si trovano cresciute e aderenti ai suoi filamenti, e che hanno l' aspetto di altrettante patelle (Apothecia), contribuiscono maggiormente a dargli una tale apparenza. Il suo colore in generale è di un verde pallidissimo. Osservata sotto la lente, $\mathrm{i}$ suoi filamenti compariscono fittamente articolati, trasparenti, pallidi, e di un bellissimo verde le loro articolazioni.

58. SPILOMA roseum: crusta crassiuscula orbiculata rosea pro aetate albida, apotheciis subrotundis sparsis vel aggregatis convexiusculis semi-immersis sanguineis. Rad. Atti della Soc. Ital. delle Sc. V. XVIII. p. 33. Tav. $1 I$. 
Comunissimo su i muri vecchi nelle vicinanze di Riojaneiro. Trovasi ancora sugl' alberi, ma raramente.

59. ARTHONIA Swartziana ( $\alpha$ ) crusta membranacea albida; apotheciis sessilibus tumidulis subrotundis repandis irregularibus elevato-punctatis atris. Achar. Lich. univers. p. 142 .?

60. LECIDEA cinereo-fusca. Ach. Lichen.univers.p. 202 ?

61. OPEGRAPHA chrysocarpa : crusta membranacea sublaevigata albo-glaucescente ac cinerascente, apotheciis variis longioribus flexuosis rugosis auratis, disco canaliculato utrinque linea longitudinali nigra notato. Rad. Atti della Soc. It. T. XVIII. p. 34. t. III. fig. 2.

62. OPEGRAPHA cylindrica: crusta tenui albo-cinerascente subnitida, apotheciis minutis elongatis cylindricis flexuosis nigris, disco clauso. Rad. M. d. Soc. It. T. XVIII. p. 34. t. III. fig. I.

63. OPEGRAPHA abnormis. Ach. Lichen. univ.p. 259?

Le cinque sunnominate specie trovansi tutte sulla scorza di diversi alberi nelle vicinanze di Mandiocca.

64. OPEGRAPHA cymbiformis: crusta tenuissima cinerascente aut nulla, apothecis cymbiformibus nigris, disco subcanaliculato planiusculo, margine elevato crenato. Nob.

È abbondantissima questa specie sulla scorza degl' alberi, che s' incontrano a due ore circa di distanza dal subborgo denominato Bota-Fogo.

Gli Apotecj di questo Lichene sono in principio lineari, ed hanno il loro disco quasi chiuso dal margine, quindi si slargano e prendono la forma d' una Barchetta: il loro disco è alquanto piano, ed ha sovente una piccola scanalatura longitudinale nel mezzo ; il margine di essi presenta una serie continovata di solchi trasversali piuttosto profondi, i quali vanno a perdersi nella parte inferiore dell' Apotecio medesimo. Accade, ma rarissimamente, che qualcuno di detti Apotecj, elevandosi alcun poco, prende una figura quasi triangolare; Spesso accade ancora, che questa specie 
trovasi affatto priva di tallo, come non di rado succede in altre ancora di questo medesimo genere.

65. GRAPHIS marginata: crusta membranacea laevigata nitida albo-glaucescente; apotheciis emergentibus flexuosis simplicibus, disco rimaeformi albo-marginato, margine thallorle elevato membranaceo lacinulato vel lacerato. Rad. Mem. della Soc. Ital. T. XVIII. p. 34. t. III. f. 3.

Sulla montagna denominata Corco-secco.

66. VERRUCARIA gemmata; crusta effusa tenui laevigata albido-incaua; apotheciis sparsis hemisphaericis papillatis nitidis, nucleo globoso hyalino. Achar. Lichen. univ. p. $27^{8}$. Schrad. Spicil. Fl. Germ. 109. t. 2. f. 3.

67. THELOTREMA lepandinum $\beta$. bahianum: crusta membranacea cinereo-virescente; apotheciorum verrucis pluribus connatis, apertura tenui punctiformi. Ach.Lichen.univ. p. 312 ?

68. LECANORA punicea : crusta tenui subpulverulenta inaequabili albida; apotheciis crassiusculis; disco plano dilute cerino puniceo marginem thallodem subcrenatum aequante. Achar. Lichen. univ. p. 395.

69. LECANORA aceroulata: crusta tenui laevigata viridi-cinerascente aut subsulphurea; apothecis in acervulis difformibus sparsis, disco convexiusculo rubro-fuscescente, margine thallode tumescente luteo flexuoso sinuato discum vix superante. Nob.

Trovasi con le tre precedenti specie sulla scorza degl' alberi nelle vicinanze di Mandiocca.

Gl' Apotecj di questo Lichene sono ordinariamente disposti in gruppetti di quattro o cinque per ciascheduno, distanti fra loro e irregolarmente sparsi sulla sua crosta, la quale è d'ordinario cenerino-verdognola, e qualche volta un poco tendente al giallognolo. Il loro disco è rosso-scuro, quasi piano in principio, convesso dipoi; il loro margine è alquanto tumido in fuori, e sopravanza appena il livello del disco medesimo; è giallo oppure di un giallo-dorato, flessuo- 
so e con dei seni più o meno profondi, i quali sovente lo sono fin quasi al centro del disco. Nel loro nascere però sono questi Apotecj per la massima parte rotondi, in particolare que' pochi che travansi solitarj.

70. STICTA crocata; thallo glauco-fuscescente sublacunoso lacero-lobato, lacinularum marginibus pulverulentis flavidis, subtus lanuginoso: sorediis minutis citrinis; apotheciis sparsis fusco-nigris. Ach. Lich. univ. p. 447 .

var. $\beta$. gilva. p. 448 .

Lichenoides lacunosum rutilum, marginibus flavis. Dill. H. Musc. tab. 84. f. I 2.

7. STICTA tomentosa; thallo laevigato glauco suborbiculari, lobis profunde incisis, lacinutis bifidis crenato-lobulatis, subtus lanuginoso : cyphellis sessilibus concavis albicantibus; apotheciis sparsis, disco plano nigro-fusco, marginem thallodem subintegrum aequante. Ach. Lich. univ. p. 450 .

72. STICT A dissecta: thallo albido-virescente lato suborbiculari lacero-laciniato, lobulis ultimis sinuatis rotundatis crenatis, subtus villoso-subvenoso; apotheciis sparsis disco concavo rubro, margine thallode crenulato inflexo. Ach. Lichen. univ. p. 45 I. Hoffm. pl. Lichen. t. 47.f. I-3.

Questa, egualmente che le due precedenti specie, trovansi in quantità sui tronchi dei diversi alberi di oui son ricuoperte tutte le montagne d' Estrella.

73. PARMELIA pannosa; thallo stellato cinereo-virescente, subtus nigro-tomentoso, laciniis planis connatis imbricatis ultimis multifidis angustis margine subelevatis; apotheciis demum convexis fuscis nigricantibus, margine integro tandem concolori subevanescente. Ach. Lichen. univ. $p$. 465 ?

Trovasi sugl' alberi nella Parrocchia (Freguezia) di S. Gio. Battista non lungi dal subborgo di Bota-Fogo.

74. BORRERA flavicans; thallo fulvo nudo ramoso, laciniis diffuso-complicatis teretiusculis compresso- subangulosis 
dichotomis flexuosis attenuatis; apotheciis submarginalibus, disco plano aurantiaco, margine thallode integro nudo. Achar. Lich. univ. p. 504.

75. BORRERA exilis; thallo exalbido compressiusculo, laciniis ramosissimis divaricatis intricatis capillaceo-attenuatis; apotheciis sparsis, disco planiusculo croceo, margine tenui integro. Achar. Lichen. univ. p. 505.

Trovansi ambedue le suddette specie su diversi alberi nei contorni di Rio-janeiro, il più frequentemente però sul Caju (Anacardium occidentale Lin. ).

76. PELTIDEA scutata $\beta$. collina; thallo subtus nudo passimque venis tomentosis nigricantibus obsesso, lobis elongatis margine lobato-crispis; apotheciis transverse oblongis subrevolutis. Achar. Lichen, univ. p. 515?

an Sp. nova.

Trovasi in abbondanza sulle montagne d'Estrella, sopra i massi, ed anche sulla nuda terra.'

77. CENOMYCE oxycera $\beta$. medusina; podetiis gracilissimis filiformibus dichotomis albis, ramis ramulisque strictis erectis adpressis furcellatis nigro-apiculatis. Achar. Lich. univ. p. 557 .

78. CENOMYCE uncialis $(\alpha)$, thallo subnullo, podetiis aggregatis albo-virescentibus, ramis brevibus patulis ad apices dilatatis subperforatis radiato-denticulatis, denticulis fuscis. Achar. Lichen. univ. p. 558.

Trovasi egualmente che la precedente specie sulle montagne d' Estrella, la prima sugl'alberi, la seconda sulla terra.

79. CENOMYCE verticillaris; podetiis tereti-compressiusculis erectis ramosis nudis albis vel albo-cinerascentibus, ramulis verticillatis; apotheciis terminalibus fuscis. Rad. Mem. della Soc. Ital. T. XVIII. p. 34. t. III . fig. 4.

Trovasi sulle più alte montagne della Provincia di Riojaneiro.

80. STEREOCAULON ramulosum; thallo albo-pallescente ramoso scabro fibrilloso, ramis sparsis elongatis sub- 
simplicibus; apotheciis terminalibus demum subglobosis fusco-nigricantibus. Achar. Lichen. univ. p. 580. Bory. Voy. 3. p. 106. $t .16 . f .3$.

E comunissima questa specie sulle montagne d' Estrella, sempre sulla nuda terra. Trovasi anche in gran copia sulle montagne di Madera.

8 I. USNEA florida; thallo erectiusculo scabrido cinereopallido fibrillis horizontalibus, ramis patentissimis expansis subsimplicibus; apotheciis planis latissimis ciliatis, ciliis radiantibus elongatis. Achar. Lichen. univ. p. 620.

Sopra i diversi alberi nelle adiacenze di Mandiocca.

Lichen capillaceus, ex cinereo rutilans, receptaculis florum subcinereis, et barbatis. Mich. Nov. pl. gen. $p \cdot 77 \cdot t .39$. fig. 5. (ottima).

82. COLLEMA azureum; thallo foliaceo membranaceo tenerrimo laevi pellucido caerulescente, lobis rotundatis glabris integerrimis; apotheciis sparsis subpodicillatis, rubris, margine pallido. Ach. Lichen. univ. p. 654. Rad. Mem. della Soc. Ital. T. XVIII. tab. IV. fig. I.

83. COLLEMA bullatum; thallo foliaceo membranaceo tenerrimo plumbeo subdiafano subrugoso granulatoque, lobis irregularibus rotundatis sinuato-laciniatis subcrenatis; apotheciis sparsis a thallo valde elevatis convexiusculis rufis, margine pallido. Achar. Lichen. univ. 655. Rad. Mem. della Soc. Ital. t. IV. fig. 2.

84. COLLEMA venustum: thallo foliaceo membranaceogelatinoso pellucido plumbeo rugoso granulatoque, lobis elongatis rotundatis, marginibus plicato-flexuosis crispis laciniatis; apothecis a thallo paullo elevatis rubris; margine rugoso foliaceo crispo. Nob.

Il tallo di questo elegantissimo Lichene tiene, nella sua forma e figura, il mezzo fra il Lichen marginellus di Swartz (Collema marginellum Ach.), e la Tremella lichenoides di Lineo (Collema lacerum Ach.), a cui somiglia ancora nel colore e grossezza. Le piccole lacinie de' suoi lobi sono ora 
rotondate, ed ora acute e fitte di mániera a rappresentare una piccola frangia. Gl' Apotecj hanno il loro margine doppiamente foliaceo, rugoso, un poco laciniato oppure crenato, e ondulato.

85. COLLEMA tremelloides $(\alpha)$ thallo foliaceo membranaceo tenerrimo subdiaphano plumbeo obsolete rugoso impressoque punctato, lobis oblougis rotundatis incisis integris; apotheciis subpodicillatis planis rubris, margine pallido. Ach. Lichen. univ. p. 655. Hoffm. Pl. Lich.t.35.f.2.

Trovasi con le altre tre precedenti specie sopra i sassi ingombrati di Musco, egualmente che su i tronchi degl'alberi nelle vicinanze di Mandiocca, e su quasi tutte le montagne d' Estrella.

86. THELEPHORA pavonia; imbricata, pileo sessili membranaceo plano reniformi fasciato; hymenio albicante. Swartz Fl. ind. occ. T. III. pag. 1930.

Ulva montana, plana reniformis, sessilis aggregata zonata, subtus incava. $S_{W}$. Prod. p. 148.

Telephora Pavonia: membrancea, tenuis, semicircularis, supra virens, sulcis concentricis, margineq!?e involuto, subtus aequabilis farinoso-glebulosa, alba. Bertol. Opusc. Sc. di Bol. p. 8. tab. 1.f. 2.

È comunissima sulle montagne, che costituiscono la cosi detta Serra degl' Organi.

87. TELEPHORA palmetto: stipitata, solitaria aut subgregaria, coriaceo-molliuscula, hymenio cuneato - flabelliformi, laciniato, supra striato-fibroso; stipite gracili compressiusculo subtomentoso.

Trovasi sulle montagne sopraindicate a piè degl'alberi, dove però è piuttosto rara.

Il suo colore è in generale d'un giallo-scuro; ha presso a poco l'abito, ovvero struttura del Fuco Palmetto, da cui differisce per non esser punto ramosa; è irregolarmente, e più o meno profondamente laciniata, ed il suo stipite, il quale, in apparenza, sembra alquanto tomentoso, ma che, 
se si osserva con una acuta lente, appare coperto di asperosità e piccole squamime biancastre.

88. CLAVARIA ceranoides: cespitosa, clavulis dentatis et obsolete ramosis subdifformibus flavescentibus apice fuscis. Pers. Syn. Fung. pag. 594?

G' individui da noi osservati differiscono-dalla ceranoide descritta dal Sig. Persoon e figurata dal Sig. Sowerby t. 235, per essere di sostanza coriacea, e alquanto tomentosa dalla base fin verso l'estremità delle sue diramazioni. Queste estrémità sono liscie tanto esternamente, che internamente, un poco più laciniate e nere. an $\mathrm{Sp}$. nov.?

89. CLAVARIA furcata: caespitosa fusca duriuscula, caulibus ramosis subdichotomis apice furcatis acutis. Nob.

an Clav. fusca. $S_{W}$. Fl. ind. occ. III $p$. 1940?

Trovasi con la precedente a piè degl' alberi sulle montagne d'Estrella. E alquanto compressa presso le ascelle o inforcazioni dei rami, e le estremità loro son sempre biforcate e acutissime," come nella Cenomyce furcata Ach. (Lichen Lin.).

90. TREMELLA foliacea: gregaria utrinque glabra tenuis undulato-plicata concava, basi crispa, cinnamomeo-carnea. Pers. Obs. myc. P. II. pag. 98?

Sugl' alberi presso Mandiocca. 


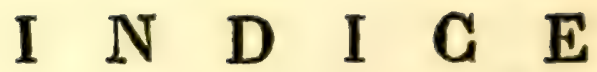

\section{Dei Generi e specie contenute nella presente Memoria.}

Anthoceros brasiliensis. Nob. Arthonia Swartziana? Achar. Borrera flavicans. Ach. exilis. Ach.

Candollea simplex. Nob. diffusa. Nob. adiantoides. Nob. cristata. Nob. repanda. Nob. complanata. Nob.

Catharinea pseudo-polytrichum. $N$. Cenomyce oxycera $\beta$. medusina. Ach.

\begin{tabular}{l} 
verticillaris. Nob. \\
uncialis (๙). Ach. \\
Collema azureum. Ach. \\
bullatum. Ach. \\
tremelloides (x). Ach. \\
venustum. Nob. \\
Conferva lichenoides. Nob. \\
Clavaria ceranoides? Pers. \\
\hline
\end{tabular}

Dicranum megalophyllum. Brid. Frullania brasiliensis. Nob. dichotoma. Nob. filicina. Nob.

Frullanoides rio-janeirensis. Nob. densifolia. Nob.

Fucus baceiferus Turn.

—— flagelliformis. Turn.

—— natans. Lin.

Graphis marginata. Nob.

Hookeria per:dula. Hook.

Hypnum circinale. Hook.

— ? longissimum. Nob.
Hypnum imbricatum. Scwägr. _ ? multiflorum. Nob.

— patulum. Schwägr. spiniforme. Hedw.

Jungermannia brasiliensis. Nob.

- connata. Swartz. pallens. $S w$. - prostrata. $S w$.

—_ 4-partita? Hook.

- Serpillifolia. Dick. - serpillifolioides. Nob. tomentella. Ehrh.

Lasia orthotrichoides. Nob.

Lecanora acervulata. Nob.

- punicea. Achar.

Lecidea cinereo-fusca? Ach.

Leskea mollis. Hedw. - rotulata. Hedw. Marchantia chenopoda. Lin.

—_ hirsuta. Swartz. papillata. Nob.

Metzgeria ciliata. Nob.

Neckera undulata. Hedw.

Octoblepharium albidum. Hediw.

Opegrapha abnormis? Ach.

chrysocarpa. Nob.

cylindrica. Nob. cymbiformis. Nob.

Orthotrichum longirostrum. Hook.

- rugifolium. Hook. Swainsoni. Hook.

Parmelia pannosa? Ach.

Peltidea scutata $\beta$. collina? Ach. Pilotrichum scabrisetum. Brid. Polytrichum glabrum. Brid. 
Pterigynandrum fulgens. Hedw. Pterogonium myurum. Hook. Racopilum tomentosum. Brid. Riccia glauca? Lin.

Schlotheimia viticulosa. Nob. Schulthesia brasiliensis. Nob. Sphagnum magellanicum. Brid. Spiloma roseum. Nob. Stereocaulon ramulosum. Ach. Sticta crocata. Ach. dissecta. Ach. tomentosa. Ach.

Thelephora pavonia. $S w$. Palmetto. Nob.

Thelotrema lepadinum? Ach.

Tremella foliacea. Pers.

Ulva undulata. Nob.

Usnea florida. Ach.

Verrucaria gemmata. Ach.

Viviania hymenophyllum. Nob. sinuata. Nob. 




Pressboard

Pamphlet

Binder

Gaylord. Bros. Inc.

Makers

Syracuse, N. $\mathbf{Z}$.

PAT. JWH 21, 1908 


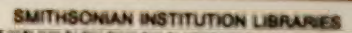

